\title{
Modeling and Simulation of High Power Ultrasonic Process in Preparation of Stable Oil-in-Water Emulsion
}

\author{
Javad Sargolzaei, Mohamad Taghi Hamed Mosavian, Attieh Hassani
}

Department of Chemical Engineering, Ferdowsi University of Mashhad, Mashhad, Iran.

Email: sargolzaei@um.ac.ir, hmosavian@gmail.com, atha2005@yahoo.com

Received March $12^{\text {th }}, 2011$; revised April 13 ${ }^{\text {th }}, 2011$; accepted April 15 ${ }^{\text {th }}, 2011$.

\begin{abstract}
The aims of this research are to study application of high power ultrasound in preparation of stable oil-in-water emulsion. The effect of $\mathrm{pH}$, ionic strength, pectin, Guar gum, lecithin, egg yolk, and xanthan gum as well as the time of sonication, temperature and viscosity of oil-water mixture on the specific surface area and size of droplets, and creaming index of the emulsion samples was investigated. The experimental data were analyzed with Taguchi method and optimum conditions were determined. In addition, an adaptive neuro-fuzzy inference system (ANFIS) was employed to modeling and categorizes the properties of the resulted emulsion. The results showed that increasing sonication time narrowed the range of droplets size distribution. Pectin and xanthan enhanced the stability of emulsion, although they had different impacts on the emulsion stability when used individually or together. Guar gum improved the viscosity of the continuous phase. Emulsions stabilized by egg yolk were found to be stable to droplet flocculation at $\mathrm{pH} 3$ and at relatively low salt concentrations.
\end{abstract}

Keywords: Ultrasound Process, Stable Oil, Egg Yolk, Emulsion, ANFIS, FIS

\section{Introduction}

Several products in food industry are oil-in-water emulsions that contain an aqueous medium with uniformly dispersed small lipid droplets such as ice cream, margarine, butter, milk, beverages, cream, dressings, dips, sauces, and desserts. [1-4]. These foodstuffs are thermodynamically unstable. For that reason, it is of importance for manufacturers to improve food emulsions stability for having no or minimal changes in the structure or consistency during storage. Size of droplets is the most important parameter in emulsion stability. This has been studied for many years leading to development of new concepts and technologies. It has long been known that ultrasound is capable of making fine emulsions [5-11]. The disintegration effect of ultrasound is due to the bubbles collapsing at the interface of two immiscible liquids disrupting one phase into another.

In this work our goal was to investigate the effect of $\mathrm{pH}$, ionic strength, pectin, Guar gum, egg yolk, xanthan gum as well as the time of sonication, viscosity of oilwater mixture and temperature on the specific surface area and size of droplets, and creaming index of the emulsion samples and create a prediction model for the emulsion properties by fuzzy modeling for employing in food industry to produce emulsion with elevated quality.

\subsection{Adaptive Neuro-Fuzzy Inference System (ANFIS) Theory}

Studies of fuzzy neural networks that combine both advantages of the fuzzy systems and the learning ability of the neural networks have been carried out. These techniques can alleviate the matter of fuzzy modeling by learning ability of neural networks and have been reported since around the beginning of 1990s. Fuzzy neural networks can be applied not only for simple pattern classification but also for meaningful fuzzy if-then rules creation; therefore, they can be put into practice for various applications. In the early stage of fuzzy neural network researches, Lin et al. $[12,13]$ proposed one of the current prima models that decide the initial fuzzy model by Kohonen's self-organizing algorithm [14] and carry out parameter adjustment by back propagation algorithm. Also as a representative example, Jang et al. proposed ANFIS [15] in 1993. ANFIS applies a neural network in deter- 
mination of the shape of membership functions and rule extraction. However, since it needs to divide the input data space in advance, accuracy of the system depends much on the achievement of this pre-processing. Wang reported an approach to acquire fuzzy rules by dividing input space [16]. These techniques, however, do not consider the output data space, so the obtained rules should not be always reasonable. Since the architecture and behavior of ANFIS are very applicable [17], it has been adopted as a basic component for interpretation researches $[17,18]$.

Normalization of inputs leads to avoidance of numerical overflows due to very large or very small weights [2]. Therefore, data are normalized by the following relationship:

$$
V_{N}=\left(1-\Delta_{U}-\Delta_{L}\right) \frac{V-V_{\min }}{V_{\max }-V_{\min }}+\Delta_{L}
$$

Where $V_{N}$ is the normalized value of $V$, the $V_{\max }$ and $V_{\min }$ are the maximum and minimum values of $\mathrm{V}$, respectively. From experience, the authors have found that a better fit will be achieved if $\Delta_{U}$ and $\Delta_{L}$ (small margins) are kept at a value of 0.05 [19].

\section{Materials and methods}

\subsection{Materials}

Pectin extracted from citrus peel, Xanthan Gum extracted from Xanthamonas campestris, Lecithin from soybean and Guar gum were purchased from Sigma Chemical Company, Germany.

Analytical grade sodium chloride $(\mathrm{NaCl})$ and acetic acid glacial $\left(\mathrm{CH}_{3} \mathrm{COOH}\right)$ was supplied from the Merck Chemical Company, Germany.

Sunflower oil and egg were obtained from local market. Deionized water was used for the preparation of all solutions.

\subsection{Preparation of Emulsion Mixture}

Oil phase was prepared by dispersing different proportions of lecithin or egg yolk, xanthan, pectin, guar gum and $\mathrm{NaCl}$ in different amount of sun flower oil and mixed for 30 minutes by a magnetic stirrer (Model VELP SCIENTIFICA s.r.l, Europe). The oil phase mixture was then mixed with distilled water adjusted to $\mathrm{pH} 3$ or 4 by glacial acetic acid. Table 1 shows proportion of each constituent in emulsion mixture for various formulas used in the experiment.

\subsection{Ultrasonic emulsification}

The aqueous and oil phase were premixed together in a beaker using a magnetic stirrer. $5 \mathrm{ml}$ aliquot of the mixture was introduced into a round bottom glass. Sonication was carried out using a Dr. Hielscher ultrasonic processor (Model UP $200 \mathrm{H}$, Germany), with the operating frequency of $24 \mathrm{KHz}$ and power output of $460 \mathrm{~W}$. A tapered titanium sonotrode of $3 \mathrm{~mm}$ in diameter was used for sonication. The tip of sonotrode was placed $1 \mathrm{~cm}$ below the surface of mixture. All samples were sonified in 0 and $10^{\circ} \mathrm{C}$ for $60,120,180$ and 240 seconds in thermostated water bath. All sonicated experiments were conducted in triplicates. One of the three samples was used to measure creaming and the other two were used for particle size measurement.

\subsection{Particle Size Measurement}

Drop size distributions of samples were measured immediately after sonication and also after one month storage at $4^{\circ} \mathrm{C}$ using Fritsch laser diffraction analyzer (Model Analysette 22, Germany).

\subsection{Viscosity Measurement}

Viscosity was measured using Brooke Field DV-II (at 80 $\mathrm{RPM}$ ) at $27^{\circ} \mathrm{C}$. The types of spindles used were S62, S63 and S64.

\subsection{Creaming Measurement}

Ten $\mathrm{ml}$ of each emulsion were transferred into a test tube, and then stored for 1month at refrigerator temperature $\left(4^{\circ} \mathrm{C}\right)$. During storage a number of emulsions separated into an optically opaque 'cream' layer at the top and a transparent (or turbid) 'serum' layer at the bottom. Creaming index was calculated by dividing the total height of cream to the total height of the column of emulsion.

\section{Statistical Analysis}

Taguchi L32 orthogonal array design was used to investigate the effect of $\mathrm{pH}$, sonication time, temperature and concentration of lecithin, egg yolk, guar gum, xanthan, pectin and sodium chloride on droplet size distribution, creaming and viscosity of samples. Table 1 shows the L32 orthogonal array design used in this study.

\section{ANFIS Design}

The ANFIS information errors used for process are shown in Table 2. There are 0.5, 1.25, 0.5, 0.15 values for ranges of influence, squash factor, accept ratio, and reject ratio. Also the optimum method is back propagation. The best threshold of the generation ANFIS is 0.05 for Egg yolk and Lecithin process simulations.

ANFIS architecture for multi input and single output (for example, viscosity) data is shown in Figure 1.

In this work, the applications of ANFIS for prediction of viscosity, creaming, arithmetic mean diameter, and specific surface area were tested at different operation conditions. For each variable, the optimum values of 
Table 1. Taguchi L-32 orthogonal array (egg yolk).

\begin{tabular}{|c|c|c|c|c|c|c|c|c|c|}
\hline \multirow{2}{*}{ Experiment number } & \multicolumn{9}{|c|}{ Factors } \\
\hline & oil & Egg yolk (gr) & Xanthan (gr) & Pectin (gr) & Guargum (gr) & $\mathrm{NaCl}$ (gr) & Time (s) & $\mathrm{pH}$ & Temperature $\left({ }^{\circ} \mathrm{C}\right)$ \\
\hline 1 & 40 & 6 & 0.1 & 0.1 & 0.1 & 0.0 & 60 & 3 & 0 \\
\hline 2 & 40 & 6 & 0.1 & 0.2 & 0.1 & 0.4 & 120 & 3 & 0 \\
\hline 3 & 40 & 8 & 0.2 & 0.1 & 0.2 & 0.8 & 180 & 3 & 0 \\
\hline 4 & 40 & 8 & 0.2 & 0.2 & 0.2 & 1.2 & 240 & 3 & 0 \\
\hline 5 & 40 & 10 & 0.3 & 0.4 & 0.4 & 0.0 & 180 & 3 & 0 \\
\hline 6 & 40 & 10 & 0.3 & 0.3 & 0.4 & 0.4 & 240 & 3 & 0 \\
\hline 7 & 40 & 12 & 0.4 & 0.4 & 0.3 & 0.8 & 60 & 3 & 0 \\
\hline 8 & 40 & 12 & 0.4 & 0.3 & 0.3 & 1.2 & 120 & 3 & 0 \\
\hline 9 & 50 & 6 & 0.2 & 0.3 & 0.4 & 0.8 & 60 & 3 & 10 \\
\hline 10 & 50 & 6 & 0.2 & 0.4 & 0.4 & 1.2 & 120 & 3 & 10 \\
\hline 11 & 50 & 8 & 0.1 & 0.3 & 0.3 & 0.0 & 180 & 3 & 10 \\
\hline 12 & 50 & 8 & 0.1 & 0.4 & 0.3 & 0.4 & 240 & 3 & 10 \\
\hline 13 & 50 & 10 & 0.4 & 0.2 & 0.1 & 0.8 & 180 & 3 & 10 \\
\hline 14 & 50 & 10 & 0.4 & 0.1 & 0.1 & 1.2 & 240 & 3 & 10 \\
\hline 15 & 50 & 12 & 0.3 & 0.2 & 0.2 & 0.0 & 60 & 3 & 10 \\
\hline 16 & 50 & 12 & 0.3 & 0.1 & 0.2 & 0.4 & 120 & 3 & 10 \\
\hline 17 & 60 & 6 & 0.3 & 0.1 & 0.3 & 0.8 & 240 & 4 & 0 \\
\hline 18 & 60 & 6 & 0.3 & 0.2 & 0.3 & 1.2 & 180 & 4 & 0 \\
\hline 19 & 60 & 8 & 0.4 & 0.1 & 0.4 & 0.0 & 120 & 4 & 0 \\
\hline 20 & 60 & 8 & 0.4 & 0.2 & 0.4 & 0.4 & 60 & 4 & 0 \\
\hline 21 & 60 & 10 & 0.1 & 0.4 & 0.2 & 0.8 & 120 & 4 & 0 \\
\hline 22 & 60 & 10 & 0.1 & 0.3 & 0.2 & 1.2 & 60 & 4 & 0 \\
\hline 23 & 60 & 12 & 0.2 & 0.4 & 0.1 & 0.0 & 240 & 4 & 0 \\
\hline 24 & 60 & 12 & 0.2 & 0.3 & 0.1 & 0.4 & 180 & 4 & 0 \\
\hline 25 & 80 & 6 & 0.4 & 0.3 & 0.2 & 0.0 & 240 & 4 & 10 \\
\hline 26 & 80 & 6 & 0.4 & 0.4 & 0.2 & 0.4 & 180 & 4 & 10 \\
\hline 27 & 80 & 8 & 0.3 & 0.3 & 0.1 & 0.8 & 120 & 4 & 10 \\
\hline 28 & 80 & 8 & 0.3 & 0.3 & 0.1 & 1.2 & 60 & 4 & 10 \\
\hline 29 & 80 & 10 & 0.2 & 0.2 & 0.3 & 0.0 & 120 & 4 & 10 \\
\hline 30 & 80 & 10 & 0.2 & 0.1 & 0.3 & 0.4 & 60 & 4 & 10 \\
\hline 31 & 80 & 12 & 0.1 & 0.2 & 0.4 & 0.8 & 240 & 4 & 10 \\
\hline 32 & 80 & 12 & 0.1 & 0.1 & 0.4 & 1.2 & 180 & 4 & 10 \\
\hline
\end{tabular}




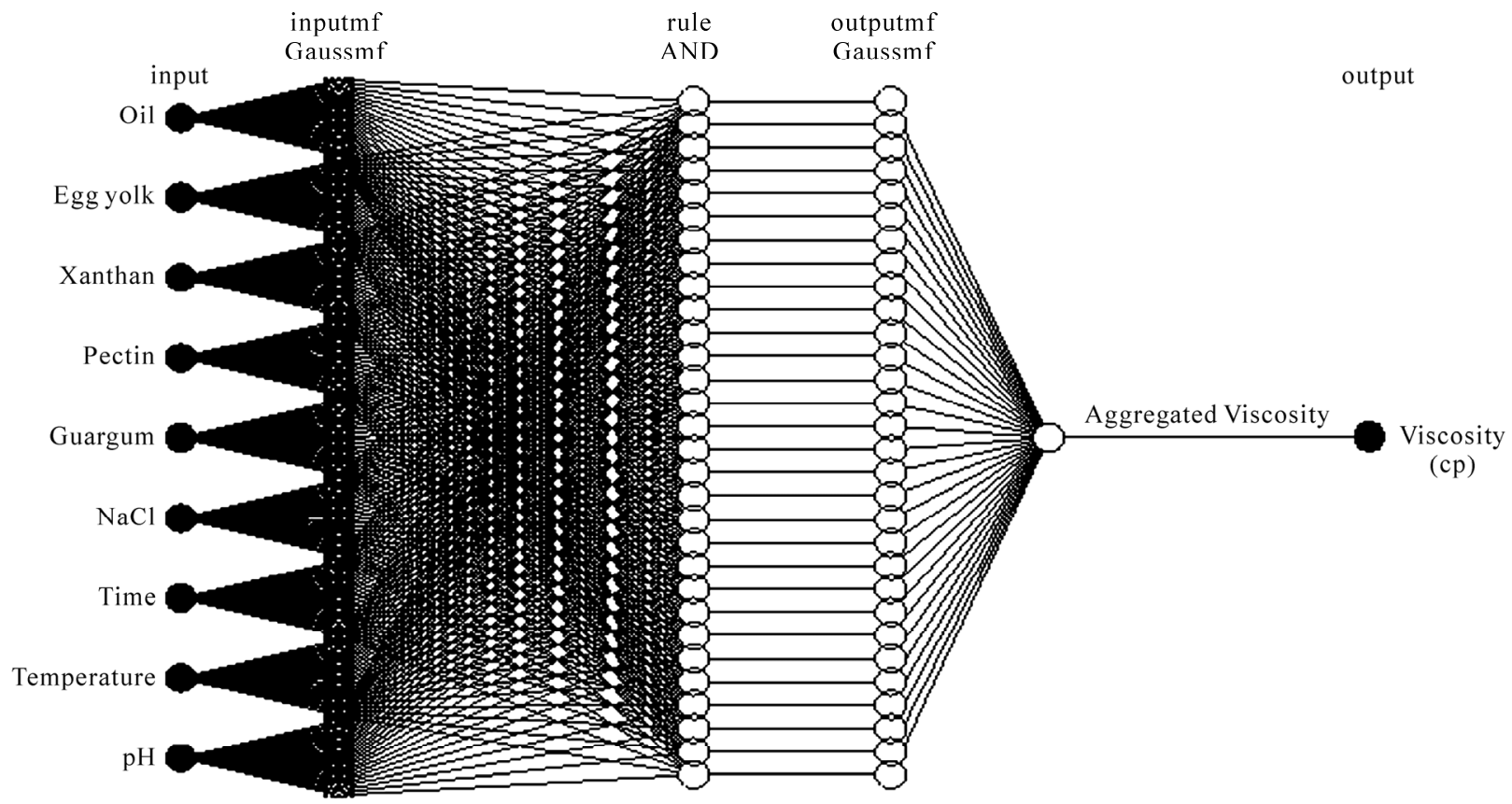

Figure 1. ANFIS Architecture of a multi-input-single-output with 30 rules in Egg yolk process.

Table 2. ANFIS information for design of network in Egg yolk with viscosity output.

Number of nodes: 612

Number of linear parameters: 300

Number of nonlinear parameters: 540

Total number of parameters: 840

Number of training data pairs: 49

Number of checking data pairs: 15

Number of fuzzy rules: 30

epoch (N optimum 100 000) were attained by minimization of root mean square error (RMSE) and SSE. It can be concluded that there is an optimum modeling using test data. Since it provides the minimum degrees of freedom sustained by testing data points. Figure 2 shows the best ANFIS is with 100000 epochs. For all output values, ANFIS is run with this epoch number. Figure $\mathbf{3}$ shows training data for viscosity of egg.

\section{Results and discussion}

\subsection{Droplet Size Distribution}

Figure 4 graphically show FIS outputs of experimental factors on arithmetic mean diameter. It is quite obvious that continuing from upper to lower levels of some factors (oil, pectin, guar gum, time, $\mathrm{pH}$, temperature) the drop
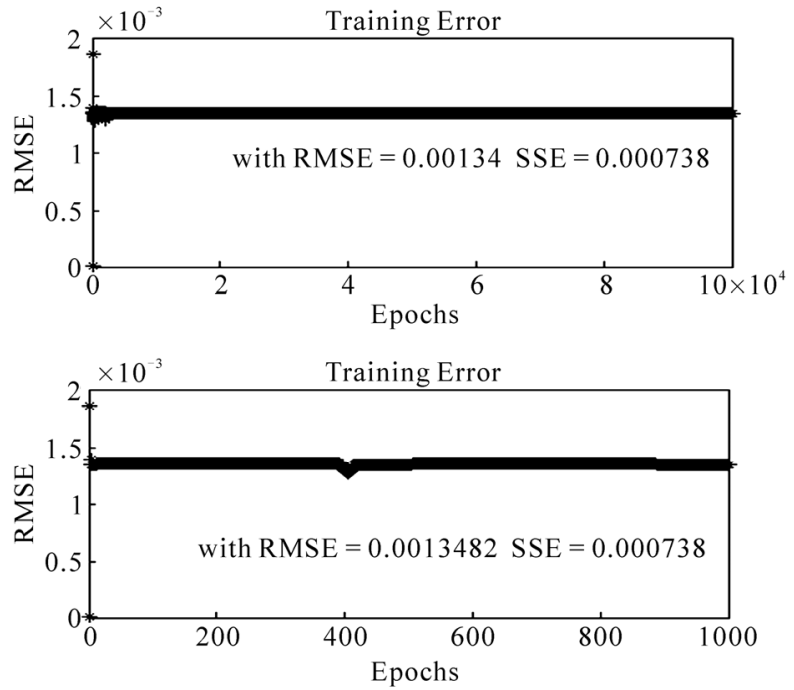

Figure 2. ANFIS training RMSE for viscosity of Egg yolk with variables input data.

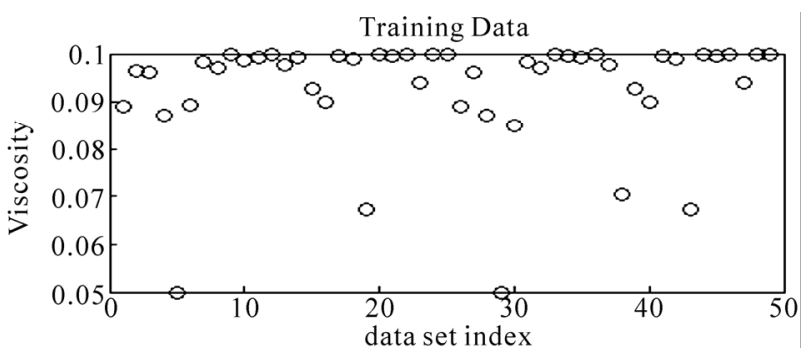

Figure 3. Shows training RMSE achieve with ANFIS for viscosity of egg. 
lets' diameter decreases. Regarding some factors (egg, xanthan, $\mathrm{NaCl}$ ), by going from upper to lower levels, droplets' diameter increases. This figure shows the higher level of oil is better for forming small mean diameter. If emulsion contains small amount of oil, aggregation phenomena will happen. In this case, other ingredient of emulsion such as emulsifiers and surfactant close each other and we have a result of particles with large mean diameter, which is not a desirable result. Oil droplet caused distance between particles and prevented flocculation and had the strongest effect on specific surface area after one month. Also higher level of $\mathrm{pH}$ is better for smaller mean diameter after sonication and after 1 month. Time of sonication had strongest effect on specific surface area in fresh emulsion. The lower level of egg yolk is better for a smaller mean diameter as an emulsifier in conjunction with surfactant such as xanthan. When the concentration of egg yolk and xanthan increased the protein of egg yolk started aggregate. Therefore according to Figure 4 small amount of egg yolk is better for a minimum particle size.

We observed protein denaturation at $10^{\circ} \mathrm{C}$ in some sample because proteins in eggs will unfold and aggregate on heating above their thermal denaturation temperature, which influences the stability and rheological properties of emulsions which is also observed by other researchers [20-22].

Emulsions stabilized by egg yolk were found to be stable to droplet flocculation at $\mathrm{pH} 3$ at relatively low salt concentrations, but unstable to flocculation at $\mathrm{pH} 3$ at high salt concentrations. A great deal of time is necessary for breaking bridging bound that may happen during sonication.

Figures $\mathbf{4}$ and $\mathbf{5}$ also shows that the effect of factors on mean diameter after one month matched earned data for fresh emulsion. The higher level of oil, guar gum, $\mathrm{pH}$, time and temperature and the lower level of $\mathrm{NaCl}$ and xanthan increased the specific surface area (Figure 5).

\subsection{Viscosity}

Acoording to Observation that obtained from FIS, we found that the viscoelasticity of emulsion was the highest at $\mathrm{pH}$ 3. The addition of medium level $\mathrm{NaCl}$ can also improve the characteristics of the emulsion, but too much $\mathrm{NaCl}$ may cause the egg yolk proteins to aggregate in the aqueous phase of the emulsion rather than forming a coating on the oil droplets [23].

In Figure 6, it was shown that the magnitude of the viscosity increases with the increase of xanthan concentration due to the interaction between emulsion droplets and xanthan gum. The viscoelasticity and stability against creaming of the emulsion should be at highest when made with a high level of egg yolk because in this way the egg yolk elements form a strong film around the lipid droplets to prevent coalescence.

\subsection{Creaming Rate}

Measurements of creaming index and observation of phase separation of the emulsions indicated that they were stable at a low level of $\mathrm{pH}$ because of adequate electro static repulsion between droplets to prevail over the attractive droplet-droplet interactions. Higher level of sonication time resulted in smaller droplets, higher viscosity and enhanced stability against creaming.

According to FIS outputs (Figure 7), using a small amount of xanthan and medium level of egg yolk increased the stability of emulsion against creaming as well as their structure by the formation of aggregates of larger

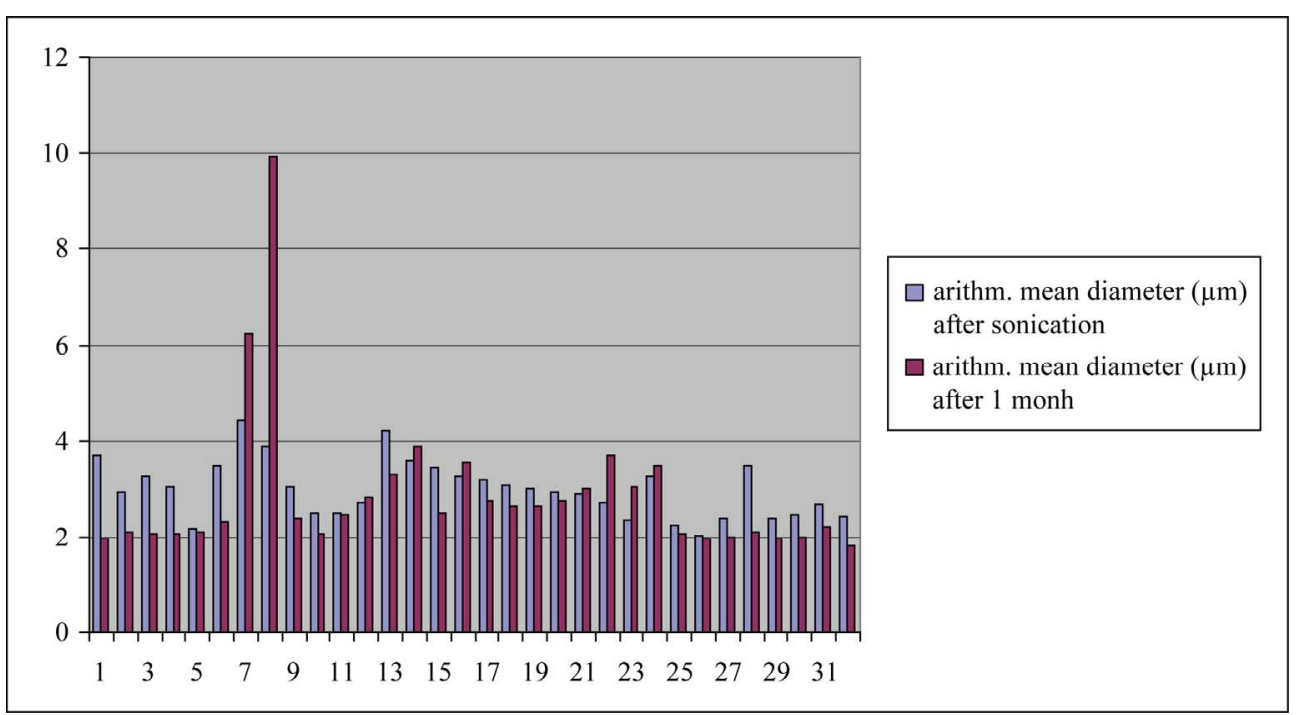

Figure 4. Arithmetic mean diameter after sonication and after 1 month for 32 samples. 


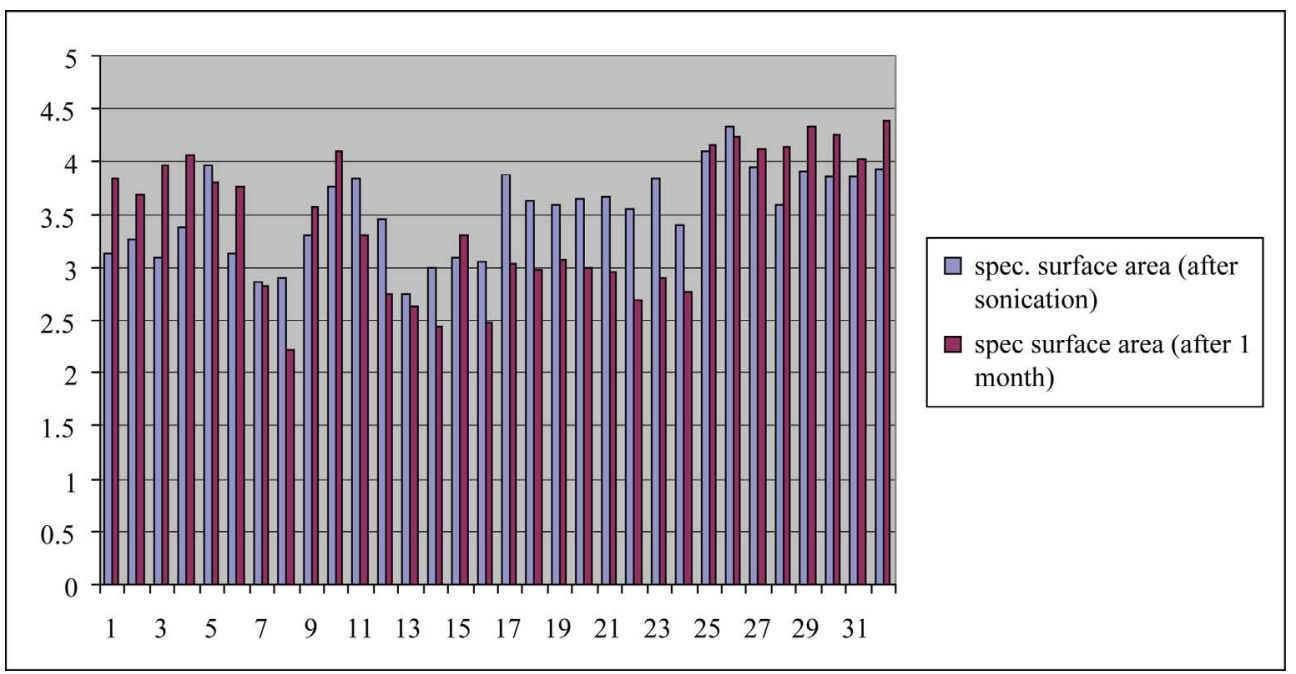

Figure 5. Specific surface area after sonication and after 1month for 32 samples.

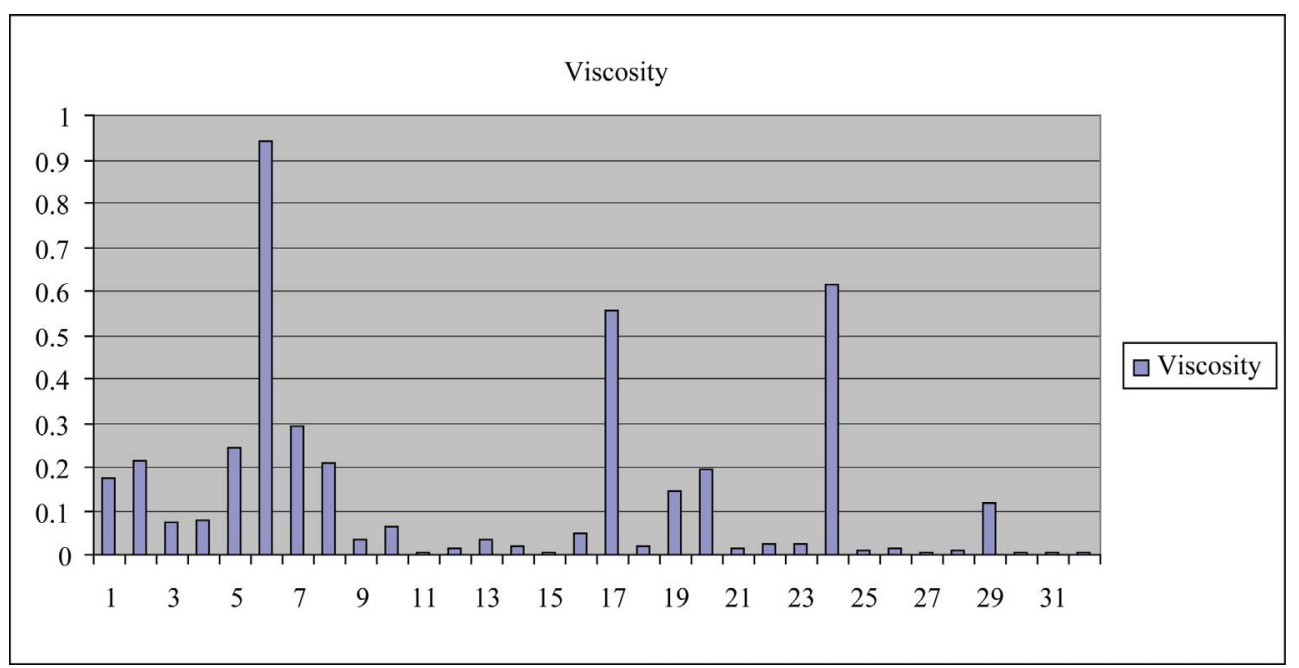

Figure 6. Viscosity of 32 emulsion formulations.

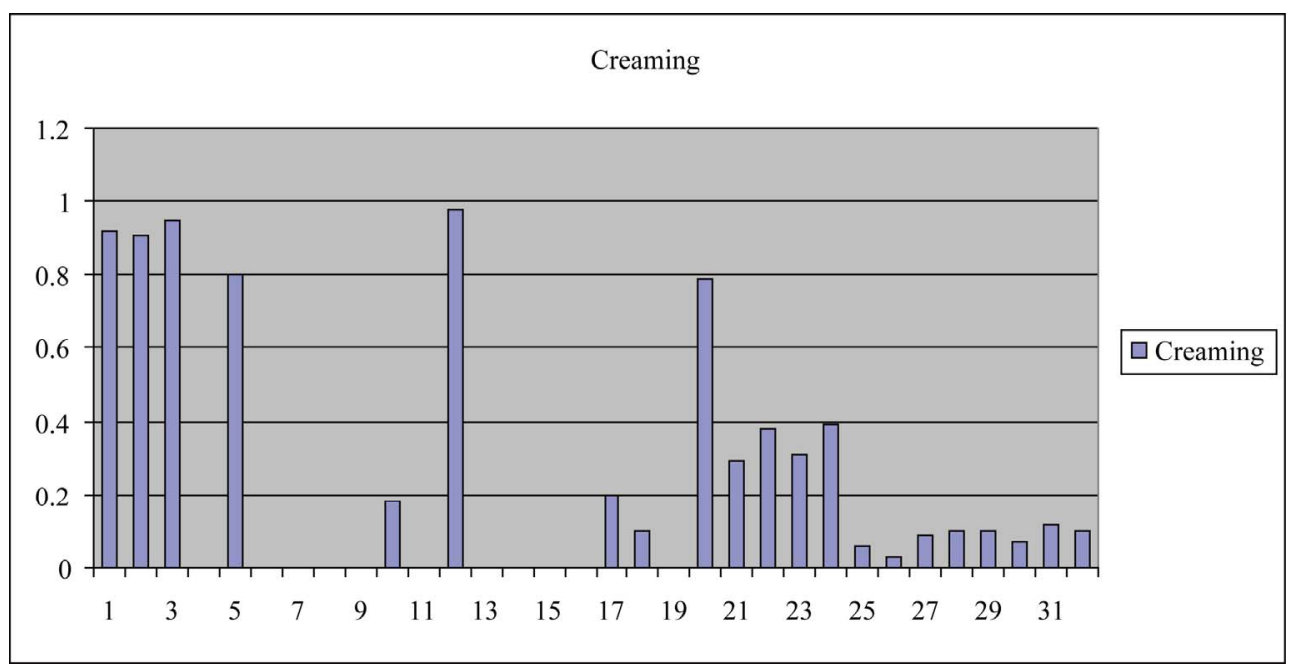

Figure 7. Creaming of 32 emulsions formulations. 
size.

\subsection{ANFIS Modeling Results}

The output values of the model are classified into two groups. The first group shows the predicted values when using input patterns belonging to train the ANFIS network, that is, near the training data set. These results allow checking the effectiveness of the model closer to the data set used for model. The second group represents the predicted values that do not belong to the training data set. These values will allow testing the model.

Figure 8 illustrates the best recall performances of ANFIS, which shows that the system is well-trained to model the actual viscosity, creaming, arithmetic mean diameter, and specific surface area.

Evidently, all plots generated by ANFIS pass through each and every training data point. All epochs in modeling are 100000 .

It is studies that the Hybrid Learning approach is supposed to converge better and faster than BP approach. The results showed that there is an excellent agreement between the ANFIS with desired data. Dynamic modeling of producing stable emulsion process performance is very important for designing and better understanding of
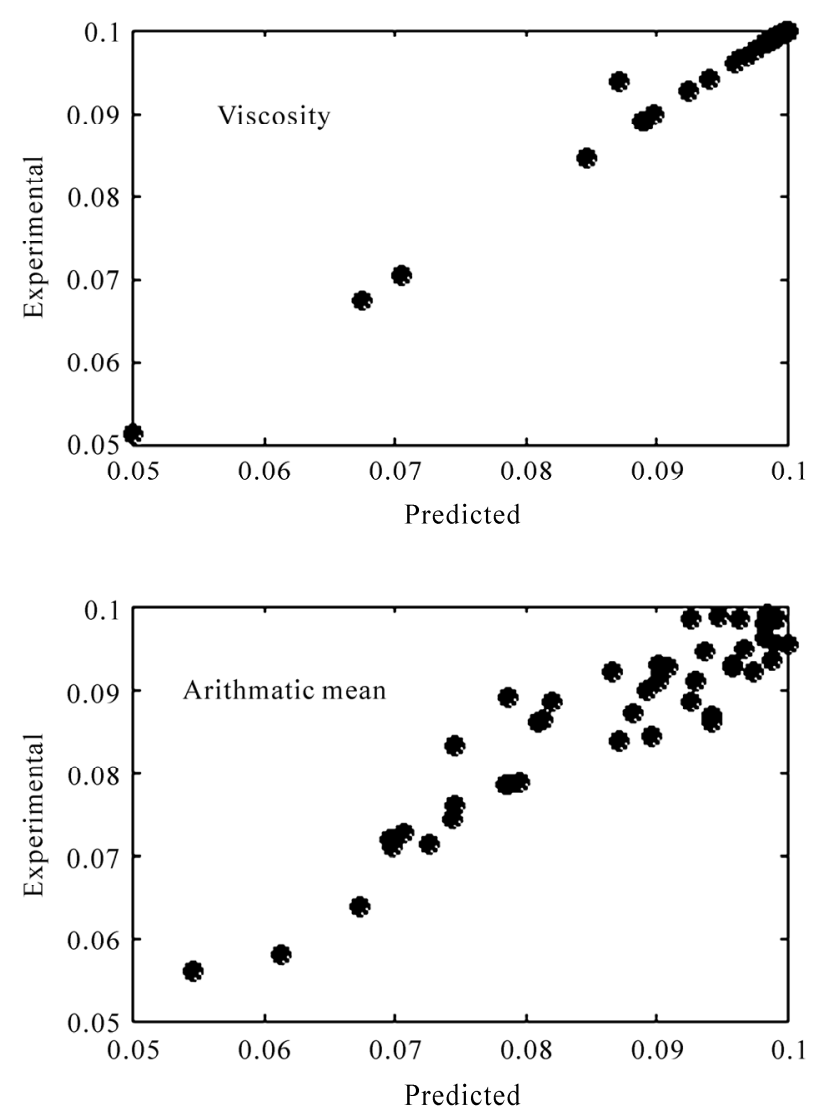

the present process. In this paper, ANFIS was applied to compare results. ANFIS approximation is able to accurately capture the non-linear dynamics of conditions that have not been used in the training process (testing data). The results showed that there is an excellent agreement between the checked data (not used in training) and modeling data, with average errors very low. As shown in Figure 9, there is agreement between the output ANFIS behavior with testing data. Also these figure shows that the ANFIS predicted values are a close match of the actual ones.

During the ANFIS training, the training set up foresaw the analytical forms of prod and probor operators for the connectors AND and OR, respectively, the min for the IF-THEN implication, the max for the ELSE aggregation, and the defuzzification method Wtaver produced the crisp output [24]. The whole procedure was implemented on a Pentium IV $330 \mathrm{MHz}$, using Matlab 7.0 (Mathworks Inc.). The ANFIS is the best manufacturer of FIS. Note that the basic fuzzy inference system (FIS) can take either fuzzy inputs or crisp inputs (which are viewed as fuzzy singletons), but outputs it produces are almost always fuzzy sets. Sometimes it is necessary to have a crisp output, especially in a situation where a fuzzy infe-
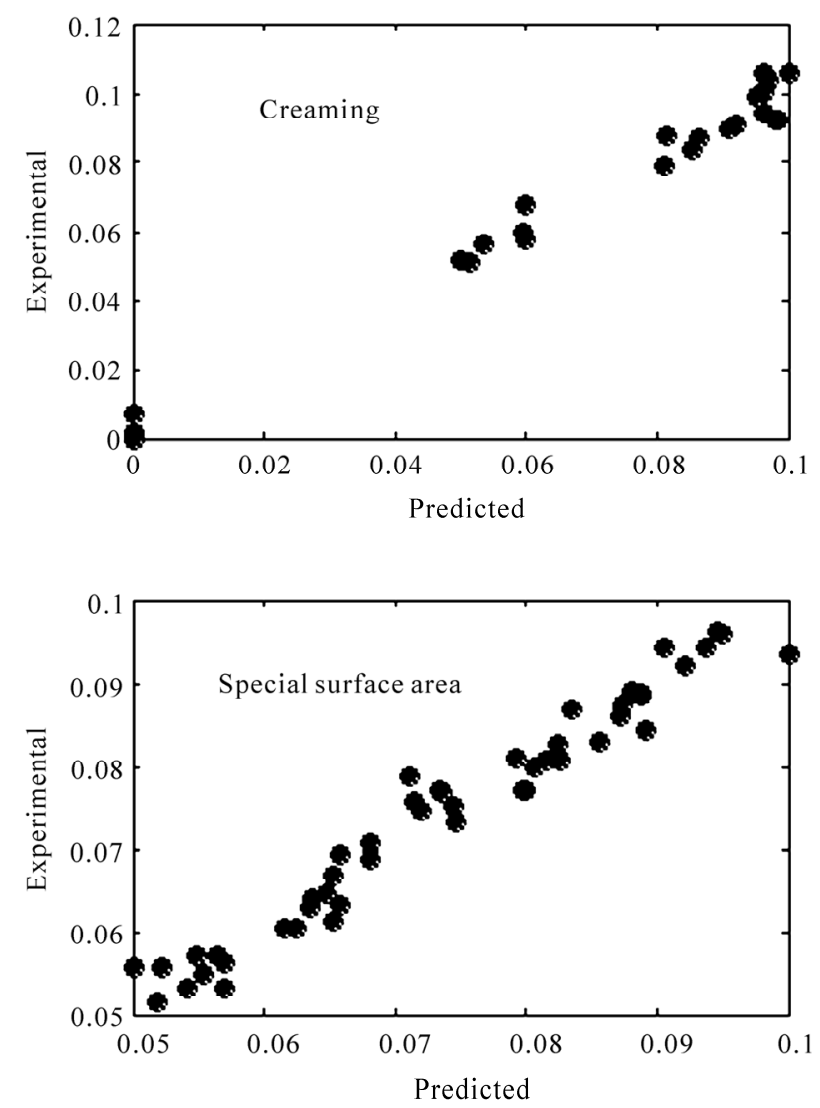

Figure 8. Comparison between values of desired and the best ANFIS for Egg yolk with training data. 

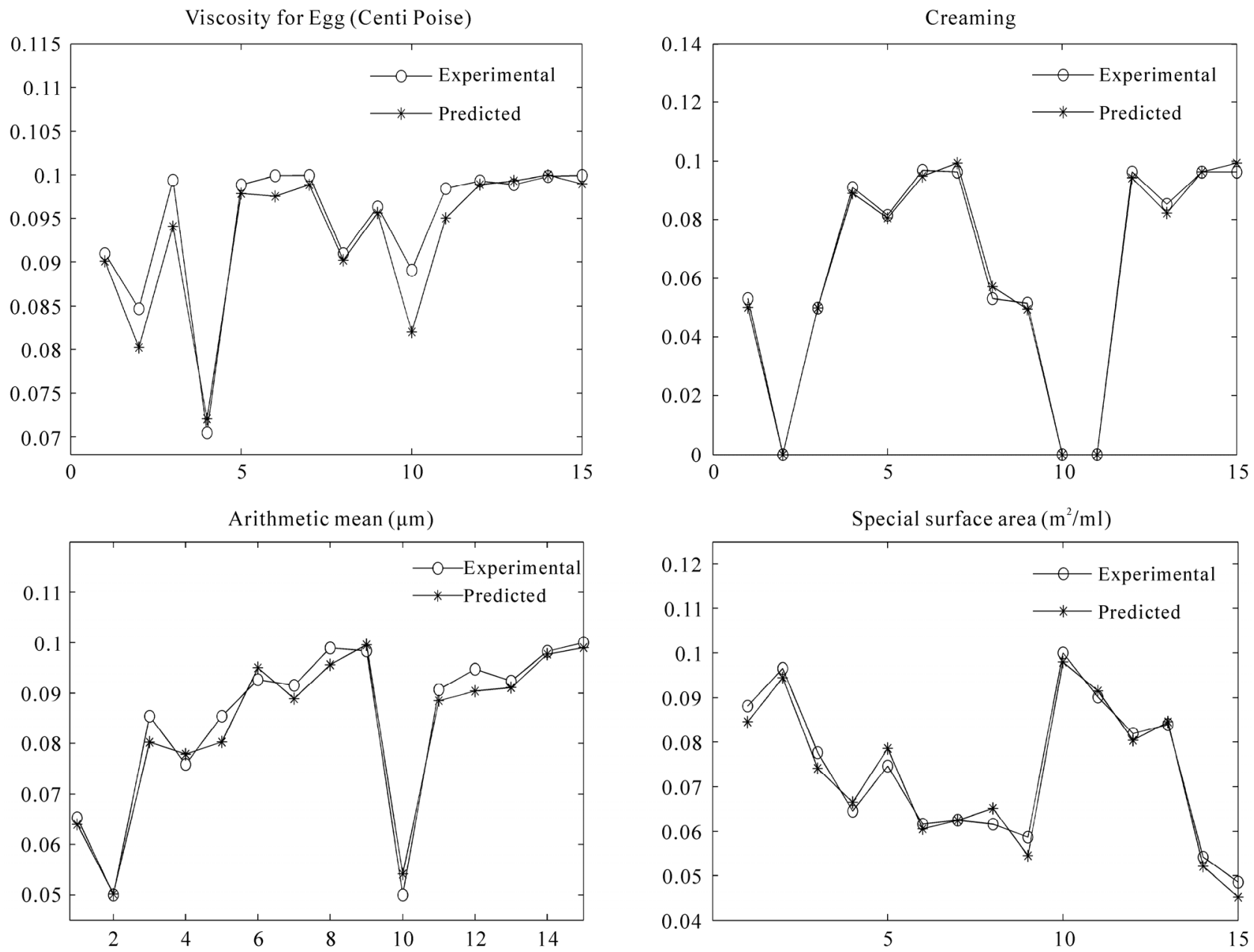

Figure 9. Comparison between values of desired and ANFIS (hybrid method) predicted of producing stable emulsion process for viscosity, Creaming, arithmetic mean diameter, and specific surface area.

rence system is used as a controller. Therefore, we need a method of defuzzification to extract a crisp value that best represents a fuzzy set. Defuzzification refers to the way a crisp value is extracted from a fuzzy set as a representative value. In general, there are several methods for defuzzifying a fuzzy set: Centroid of area (COA), Bisector of area (BOA), Mean of maximum (MOM), Smallest of maximum (SOM), Largest of maximum (LOM), and weighted average (Wtaver). These defuzzification operations are not easily subject to rigorous mathematical analysis, so most of the studies are based on experimental results [24]. Since each rule has a crisp output, the overall output is obtained via weighted average (Wtaver), thus avoiding the time consuming process of defuzzification required in a Mamdani model. In practice, the Wtaver operator is sometimes replaced with the weighted sum (Wsum) operator to reduce computation further, especially in the training of a fuzzy inference system.

\section{Conclusions}

The diameter, specific surface area of droplet, viscosity and creaming index of emulsion are strongly affected by the amount of oil. lower level of oil is better for stability and viscosity of emulsion. The existence of guar gum had supportive effect on oil behavior and contributes desirable texture. Xanthan was found to have synergic effect on pectin in stabilizing the emulsion especially at $\mathrm{pH} 4$ for specific surface area and mean diameter and $\mathrm{pH} 3$ for viscosity and creaming index. The presence of salt was shown to have positive effect on the viscosity and stability through lowering creaming index. Using high temperature was good for mean and specific surface area and low temperature preferable for smaller creaming index. High level of sonication time has a preference for egg yolk stabilized emulsion. The results of Particle size measurement showed larger specific surface area and smaller arithmetic mean diameter after 1 month. High 
level of sonication time has a preference for egg yolk stabilized emulsion.

The current study proves that ANFIS is a technique that can be used capably to predict the food properties. It is consider that this technique can be useful to predict many other peroperties and parameters in food industry.

\section{Acknowledgments}

The authors would like to thank the Ferdowsi University of Mashhad for supporting this research (P. No. 9085, 15 November 2009).

\section{REFERENCES}

[1] E. Dickinson, "Hydrocolloids as Emulsifiers and Emulsion Stabilizers,” Food Hydrocolloids, Vol. 23, No. 6, 2009, pp. 1473-1482. doi:10.1016/j.foodhyd.2008.08.005

[2] B. Abismaïl, J. P. Canselier, A. M. Wilhelm, H. Delmas and C. Gourdon, "Emulsification by Ultrasound: Drop Size Distribution and Stability," Ultrasonics Sonochemistry, Vol. 6, No. 1-2, 1999, pp. 75-83.

[3] S. O. Agboola, H. Singh, P. A. Munro, D. G. Dalgleish and A. M. Singh, "Stability of Emulsions Formed Using Whey Protein Hydrolysate: Effects of Lecithin Addition and Retorting," Journal of Agricultural and Food Chemistry, Vol. 46, No. 5, 1998, pp. 1814-1819. doi:10.1021/jf970913l

[4] J. M. Aguilera and H. G. Kessler, "Properties of Mixed and Filled-Type Dairy Gels,” Journal of Food Science, Vol. 54, 1989, pp. 1213-1221.

[5] A. Koocheki, R. Kadkhodaee, S. A. Mortazavi, F. Shahidi and A. R. Taherian, "Influence of Alyssum Homolocarpum Seed Gum on the Stability and Flow Properties of O/W Emulsion Prepared by High Intensity Ultrasound," Food Hydrocolloids, Vol. 23, No. 8, 2009, pp. 24162424. doi:10.1016/j.foodhyd.2009.06.021

[6] A. E. Alegria, Y. Lion, T. Kondo and P. Riesz, "Sonolysis of Aqueous Surfactant Solutions. Probing the Interfacial Region of Cavitation Bubbles by Spin Trapping," Journal of Physical Chemistry, Vol. 93, No. 12, 1989, pp. 4908-4913. doi:10.1021/j100349a046

[7] D. M. W. Anderson, "Gums and Stabilisers for the Food Industry 3,” Elsevier Applied Sciences, London, 1986.

[8] M. Anton and G. Gandemer, "Effect of pH on Interface Composition and on Quality of Oil-in-Water Emulsions Made with Hen Egg Yolk," Colloids and Surfaces B: Biointerfaces, Vol. 12, No. 3-6, 1999, pp. 351-358. doi:10.1016/S0927-7765(98)00089-7

[9] I. Aroutiounov, B. Toumanian, V. Melikian, D. Kovalenko and V. Zubov, "Polymerisation in Miniemulsion," 2e Congre's Mondial de l'Emulsion, Bordeaux, 23-26 September 1997, pp. 102-106.

[10] M. O. J. Azzam and R. M. Omari, "Stability of Egg White-Stabilized Edible Oil Emulsions Using Conductivity Technique,” Food Hydrocolloids, Vol. 16, No. 2, 2002, pp. 105-110. doi:10.1016/S0268-005X(01)00068-6

[11] A. A. Baram, "Soviet Physics," Acoustics, Vol. 10, No. 4,
1965, pp. 343-346.

[12] C. T. Lin and C. S. G. Lee, "Neural-Network-Based Fuzzy Logic Control and Decision System,” IEEE Transactions on Computers, Vol. 40, No. 12, 1991, pp. 13201336. doi:10.1109/12.106218

[13] A. Amano and T. Arisuka, "On the Use of Neural Networks and Fuzzy Logic in Speech Recognition," Proceedings of the International Joint Conference on Neural Networks, Washington, 1989, pp. 301-305. doi:10.1109/IJCNN.1989.118595

[14] T. Kohonen, “The Self-Organizing Map,” Proceedings of the IEEE, Vol. 78, No. 9, 1990, pp. 1464-1480. doi:10.1109/5.58325

[15] J. S. R. Jang, “ANFIS: Adaptive-Network-Based Fuzzy Inference System,” IEEE Transactions on Systems, Man and Cybernetics, Vol. 23, No. 3, 1993, pp. 665-685. doi:10.1109/21.256541

[16] L. X. Wang, “Training of Fuzzy Logic Systems Using Nearest Neighborhood Clustering," Proceedings of the 2nd IEEE International Conference on Fuzzy Systems 1, San Francisco, 1993, pp. 93-100.

[17] Anon, “Adaptive Fuzzy Inference Neural Network,” 2010. http://www.elsevier.com/locate/patcog

[18] H. Iyatomi and M. Hagiwara, "Knowledge Extraction from Scenery Images and the Recognition Using Fuzzy Inference neural Networks,” Pattern Recognition, Vol. 35, No. 8, 2002, pp. 1793-1806.

[19] J. Sargolzaei and A. Kianifar, "Modeling and Simulation of Wind Turbine Savonius Rotors Using Artificial Neural Networks for Estimation of the Power Ratio and Torque," Simulation Modelling Practice and Theory, Vol. 17, No. 7, 2009, pp. 1290-1298. doi:10.1016/j.simpat.2009.05.003

[20] M. L. Denmat, M. Anton and G. Gandemer, "Protein Denaturation and Emulsifying Properties of Plasma and Granules of Egg Yolk as Related to Heat Treatment," Journal of Food Science, Vol. 64, No. 2, 1999, pp. 194197. doi:10.1111/j.1365-2621.1999.tb15863.x

[21] J. E Moros, J. M. Franco and C. Gallegos, "Rheological Properties of Cholesterol-Reduced, Yolk-Stabilized Mayonnaise," Journal of the American Oil Chemists' Society, Vol. 79, No. 8, 2002, pp. 837-843.

[22] J. E. Moros, J. M. Franco and C. Gallegos, "Rheology of Spray-Dried Egg Yolk-Stabilized Emulsions,” International Journal of Food Science and Technology, Vol. 37, No. 3, 2002, pp. 297-307. doi:10.1046/j.1365-2621.2002.00570.x

[23] V. D. Kiosseoglou and P. Sherman, "Influence of Egg Yolk Lipoproteins on the Rheology and Stability of Oil/ Water Emulsions and Mayonnaise 1. Viscoelasticity of Groundnut Oil-in-Water Emulsions and Mayonnaise," Journal of Texture Studies, Vol. 14, No. 4, 1983, pp. 397417.

[24] J. Sargolzaei, M. Khoshnoodi, N. Saghatoleslami and M. Mousavi, "Fuzzy Inference System to Modeling of Crossflow Milk Ultrafiltration,” Applied Soft Computing Journal, Vol. 8, No. 1, 2008, pp. 456-465. doi:10.1016/j.asoc.2007.02.007 